\title{
INCIDENCE OF LYMPH NODE METASTASES IN LOCALLY ADVANCED CERVICAL CANCER IN FIGO STAGE IB2-IIB - THE ROLE OF PREOPERATIVE RADIOTHERAPY
}

\author{
Ismail E., Ya. Kornovski, S. Ivanov \\ Clinic of Gynecology, Department of Obstetrics and Gynecology, \\ St. Anna University Hospital of Varna, Medical University of Varna
}

\begin{abstract}
This study aims at determining the incidence rate, number, location and type of lymph node metastases in patients with cervical cancer in stage IB2 and IIB as classified by FIGO prior to and after radiotherapy. Between 2003 and 2011, 184 patients with cervical cancer in FIGO clinical stage IB2-IIB were operated on in the Clinic of Gynecology, St. Anna University Hospital of Varna. Surgery consisted in radical hysterectomy type III and LEP procedure in most cases. Radiotherapy was performed either preoperatively 30 Gy TGT and postoperatively until 52 Gy, or postoperatively - 52 Gy TGT. All the patients were divided into 4 groups according to the stage and order of procedures: group one - 83 patients in stage IB2 with primary surgery; group two - 11 patients in stage IB2 with preoperative radiotherapy and surgery; group three - 31 patients in stage IIB with primary surgery, and group four - 50 patients in stage IIB with preoperative radiotherapy and surgery. The incidence rate of lymph node metastases in these groups was $37,3 \%, 9 \%, 38,7 \%$, and $22 \%$, respectively. The incidence rate of macrometastases in lymph nodes in these groups was $20,4 \%, 9 \%, 25,8 \%$, and $12 \%$, respectively. Preoperative radiotherapy reduced the incidence rate of lymph node metastases in locally advanced cervical cancer in FIGO stage IB2-IIB. In these patients, lumbosacral, pre-sacral, and para-aortic lymph node dissection should be performed alongside the pelvic lymph node dissection to minimize the risk of distant metastases. The lymph node dissection occupied a central place in the surgical treatment of these patients. In addition to its therapeutic effects, it played a staging role that determined the most appropriate adjuvant treatment.
\end{abstract}

Key words: cervical cancer, FIGO stage IB2-IIB, lymph node metastases, lymph node dissection, radiotherapy

\section{INTRODUCTION}

Lymph node metastasis (LNM) is the most important post-treatment prognostic factor for patients in stage IIB-IV of locally advanced cervical cancer (LACC). The five-year survival rates for operated patients without LNM are $82 \%-90 \%$, while for those with LNM - between $38 \%$ and $61 \%$ (1). Because of the prognostic significance of LNM, pelvic lymph node dissection (PLND) should be an integral and standard part of the surgical treatment of LACC in stages IB2-IIB. Some authors propose the lymph node dissection (LND) and express histological testing of para-aortic lymph nodes (PALN) as the first step in PLND. When PALN are affected by metastases, radical hysterectomy is not performed any more (3).

It is recommended that PLND be performed with accuracy, because the larger the number of removed lymph nodes, the higher the percentage of metastases. The optimal number of re-

\footnotetext{
Address for correspondence:

E. Ismail, Clinic of Gynecology, Dept. of Obstetrics and Gynecology,

Medical University of Varna,

55 Marin Drinov Str., 9002 Varna

e-mail: elibet@abv.bg
}

moved lymph nodes must amount to 23-28. In cases of cervical cancer in stages IB2-IIB, an incidence rate of LNM above $27 \%$ is reported. According to some authors, the incidence rate of LNM is $10,5 \%$ when the removed lymph nodes are up to 20 and reaches up to $27-30 \%$ when the removed lymph nodes are more than 40-50. LND is considered optimal when the removed lymph nodes are over $20(3,4)$. In recent years, many researchers have proposed performing laparoscopic or extraperitoneal lymphatic dissections in order to detect LNM and avoid radical hysterectomy (2). Several pilot studies have lately confirmed the effectiveness of sentinel lymph node biopsy. If the sentinel lymph node proves metastatic, combined chemotherapy and radiotherapy has been advocated (3). Most recently, express histological examination of the lymph nodes along the course of the common iliac artery represents another modern innovation in the surgical treatment of LACC. If these prove metastatic, the extension of the lymphatic dissection into the para-aortic area is recommended $(4,13)$.

A number of questions concerning the approach to further treatment arise in the case of intraoperative findings of increased PALN. The irradiation of the para-aortic field achieves long-term survival of $30 \%-40 \%$ of the female patients in stage IIB with PALN. The results from a randomized trial show that in women with cervical cancer and PALN, the competing treat- 
Incidence of lymph node metastases in locally advanced cervical cancer in FIGO stage IB2-IIB - the role ...

ment with combined chemotherapy and radiotherapy based on Cis-Platina and irradiation of the para-aortic field leads to a significantly longer survival rate (4). The treatment of tumoUrs in stage IB2 and IIB stands as a real challenge regardless of the initial approach, because recurrences are more frequent than those observed in stage IB1 and IIA1. Depending on the risk level (either high, or medium), for stage IB1 and IIA1 some clinics give preference to primary surgical treatment and the respective postoperative radiotherapy/combined chemotherapy and radiotherapy, as this is the standard for stage IB1 $(5,9)$. Other authors opt for primary radiotherapy and subsequent surgery. At presence, the application of neo-adjuvant chemotherapy followed by radical hysterectomy emerges as a new alternative (2). A study of GOG 99554 is about to be completed comparing the survival rate in cases with neo-adjuvant chemotherapy + surgery versus combined chemotherapy and radiotherapy in stage IB2 as well as in stage IIB. Neo-adjuvant chemotherapy aims at reducing the volume of the primary tumour and thus creating preconditions for radical surgery. The advantage of the neo-adjuvant chemotherapy + surgery approach prior to the combined chemotherapy and radiotherapy is in the removal of the uterus and the primary tumour, as well as in lymph status assessment (6-8, 10). For comparison, when radiotherapy alone is applied in stage IB2, the recurrence rate in the pelvic area is $30 \%$, and that in the cases of primary surgery and postoperative radiotherapy $20 \%$ (14). Nevertheless, no standard method (methods) of treatment exists for stage IB2, and the treatment failure often results from the high LNM percentage. The overall 5-year survival rate for any stages in the case of lymph node negative cancer is $92,1 \%$, and in the case of lymph node positive one- $64,1 \%$ (14). Our study aims at determining the incidence rate, number, location, and type of LNM in patients with cervical cancer in stage IB2 and IIB as classified by FIGO before and after radiotherapy.

\section{MATERIAL AND METHODS}

During the period 2003-2011, 184 female patients with cervical cancer in FIGO clinical stage IB2-IIB were operated on at the Clinic of Gynecology, , St. Anna University Hospital of Varna. According to the therapy applied (other than surgery), patients' distribution is illustrated in Table 1.

The patients in stage IB2 and IIB were divided into 4 groups according to the stage and order of surgery and radiotherapy procedures: group one - 83 patients in stage IB2 with primary surgery; group two - 11 patients in stage IB2 with preoperative radiotherapy and surgery; group three - 31 patients in stage IIB with primary surgery, and group four - 50 patients in stage IIB with preoperative radiotherapy and surgery. Patients' distribution according to the surgery performed was the following: radical hysterectomy of class III with adnexa + pelvic LND 130; radical hysterectomy of class III with adnexa + pelvic LND + para-aortic LND - 8; radical hysterectomy of class III without adnexa + pelvic LND + para-aortic LND -15; laterally expanded parametrectomy (LEP) - 24; laparoscopic adnexectomy + pelvic LND - 4, and exploratory laparotomy + pelvic LND - 3 patients.
Table 1. Patients' distribution according to the applied treatment

\begin{tabular}{||l|c|c||}
\hline \hline \multirow{2}{*}{ Treatment } & \multicolumn{2}{|c||}{ Patients } \\
\cline { 2 - 3 } & $\mathrm{n}$ & $\%$ \\
\hline surgery + radiotherapy & 114 & 61,96 \\
\hline radiotherapy + surgery + radiotherapy & 61 & 33,15 \\
\hline $\begin{array}{l}\text { neoadjuvant chemotherapy + surgery + } \\
\text { radiotherapy }\end{array}$ & 6 & 3,26 \\
\hline $\begin{array}{l}\text { neoadjuvant chemotherapy + radiotherapy } \\
\text { + surgery }\end{array}$ & 3 & 1,63 \\
\hline total & 184 & 100,00 \\
\hline \hline
\end{tabular}

LEP was carried out since May, 2010 onwards in stage IIB patients after radiotherapy or without prior to radiotherapy as the main target group. With the adnexa kept intact and their transposition above the pelvis directed to the lateral canal, biopsy was performed on the PALN (in order to exclude the instance of metastasis, which requires the irradiation of the para-aortic field and renders unnecessary the transposition and preservation of the ovaries).

The enlarged, palpable lymph nodes gave indication of para-aortic LND. Laparoadnexectomy with LND was performed after radiotherapy in these patients who were, in an intraoperative way, inoperable with respect to radical hysterectomy.

LND was performed intraperitoneally in 170 patients and extraperitoneally in 14 ones. It was of a total pelvic type along the common internal and external vessels, fossa obturatoria and pre-sacral lymph nodes bilaterally. Radiotherapy was TGT in doses of 30 Gy preoperatively and with postoperative dose supplement up to $52 \mathrm{~Gy}$, or with the entire dose of 52 Gy administered postoperatively.

Data were collected from medical histories, patient's records, and the information system of the Clinic of Oncology of Varna. Their statistical processing was carried out by using of SPSS 13,0 and MedCalc 11,6 software package.

\section{RESULTS}

Tables 2 and 3 summarize LNMM frequency by group, as well as the correlation between various LNM characteristics (macroand micrometastasis, number of LNM, pelvic and para-aortic) and the four groups of stage IB2 and IIB patients.

Table 2. Presence of LNM in different patients' groups

\begin{tabular}{||l|c|c|c||}
\hline Patients' groups & $\mathbf{1 - 3}$ LNM & LMN >3 & LNM (total) \\
\hline group one $(\mathrm{n}=83)$ & $26(31,3 \%)$ & $5(6 \%)$ & $31(37,3 \%)$ \\
\hline group two $(\mathrm{n}=11)$ & $1(9 \%)$ & - & $1(9 \%)$ \\
\hline group three $(\mathrm{n}=31)$ & $8(25,8 \%)$ & $4(12,9 \%)$ & $12(38,7 \%)$ \\
\hline group four $(\mathrm{n}=50)$ & $8(16 \%)$ & $3(6 \%)$ & $11(22 \%)$ \\
\hline \hline
\end{tabular}


Table 3. LNM location and type in different patients' groups

\begin{tabular}{||l|c|c|c|c||}
\hline \hline $\begin{array}{l}\text { Patients' } \\
\text { groups }\end{array}$ & Pelvic & Para-aortic & $\begin{array}{c}\text { Micro- } \\
\text { metastasis }\end{array}$ & $\begin{array}{c}\text { Macro- } \\
\text { metastasis }\end{array}$ \\
\hline $\begin{array}{l}\text { group one } \\
(\mathrm{n}=83)\end{array}$ & $27(32,5 \%)$ & $4(4,8 \%)$ & $14(16,9 \%)$ & $17(20,4 \%)$ \\
\hline $\begin{array}{l}\text { group two } \\
(\mathrm{n}=11)\end{array}$ & $1(9 \%)$ & - & - & $1(9 \%)$ \\
\hline $\begin{array}{l}\text { group three } \\
(\mathrm{n}=31)\end{array}$ & $12(38,7 \%)$ & - & $4(12,9 \%)$ & $8(25,8 \%)$ \\
\hline $\begin{array}{l}\text { group four } \\
(\mathrm{n}=50)\end{array}$ & $8(16 \%)$ & $3(6 \%)$ & $5(10 \%)$ & $6(12 \%)$ \\
\hline \hline
\end{tabular}

\section{DISCUSSION}

Upon examination of LNM incidence rate and patterns in these four groups, i. e. in stage IB2 and IIB, the following results were established:

- Despite the high LNM incidence rate in stage IB2 and IIB (group two and group four, i. e. after radiotherapy), the incidence rates of both LNM as a whole and of macro-metastatic lymph nodes decreases.

- When comparing the group one and group two (stage IB2), LNM are 37,3\% and 9\%, respectively $(\mathrm{p}<0,001)$, while macroscopic LNM are $20,4 \%$ and $9 \%$, respectively $(\mathrm{p}<0,005)$.

- The comparison of the group three and group four (stage IIB) shows that LNM are $38,7 \%$ and $22 \%$, respectively $(\mathrm{p}<0,005)$, while macroscopic LNM are $25,8 \%$ and $12 \%$, respectively $(\mathrm{p}<0,001)$.

Despite this LNM reduction in the wake of radiotherapy, there were no significant differences in the survival rates, both overall and disease-free, among the patients from group one and group two, on the one hand, and from group three and group four, on the other hand $(11,12)$. This could be explained by the presence of micro-metastases above the true pelvis, an area which escapes the effects of irradiation. However, these metastases are a prerequisite for distant metastases. These facts demonstrate the importance of para-aortic and lumbosacral LND in cases of stage IB2-IIB cervical cancer. It should be noted that the EORTC 99554 study, the results of which are eagerly expected by the oncogynecologists in Europe, will shed more light on the treatment of cervical cancer patients in stages IB2 and IIB. In conclusion, preoperative radiotherapy reduces the incidence rate of LNM in LACC in FIGO stage IB2-IIB. In these patients, lumbosacral, pre-sacral, and para-aortic LND should be performed alongside the PLND to minimize the risk of distant metastases. LND occupies a central place in the surgical treatment of these patient groups. In addition to its therapeutic effects, it has a staging role, which determines the most appropriate adjuvant treatment.

\section{REFERENCES}

1. Committee on Practice Bulletins-Gynecology. ACOG practice bulletin. Diagnosis and treatment of cervical carcinomas, No 35, May 2002.- Obstet. Gynecol., 99, 2002, No 5, Pt 1, 855-867.

2. Eife1, P. J., J. Curtin. Early-stage cervical cancer. Gynecologic cancer. Controversies in management. Elsevier, Churchill Livingstone, 2004, 161-174.

3. Frumovitz, M., P. T. Ramirez, C. F. Levenback. Lymphatic mapping and sentinel lymph node detection in women with cervical cancer.Gynecol. Oncol., 110, 2008, No 3, Suppl. 2, S17-S20.

4. Kesic, V. Management of cervical cancer.- Eur. J. Surg. Oncol., 32, 2006, No 8, 832-837.

5. Kornovski, Y., G. Gorchev. Therapeutic options for cervical cancer after radical hysterectomy.Oncologos, 2002, 3-4, 27-29 (in Bulgarian).

6. Kornovski, Y., R. Yaneva, G. Gorchev, H. Tsekov, S. Uzunov, S. Filev. Neo-adjuvant chemotherapy for locally advanced squamous cervical cancer (stage IIIB-IVA).- Akush. ginekol. (Sofia), 2002, No 4, 39-44 (in Bulgarian).

7. Kornovski, Y., R. Yaneva, G. Gorchev, H. Tsekov, S. Uzunov, S. Filev. Combined chemotherapy before conventional local treatment (surgery and radiotherapy for cervical cancer (stage IIB-IIIB) - preliminary results.- Akush. ginekol. (Sofia), 2003, No 2, 24-31 (in Bulgarian).

8. Kornovski, Y., G. Gorchev. Neoadjuvant chemotherapy followed by radical surgery and radiotherapy vs. pelvic irradiation in cervical cancer patients FIGO stage IIB-IVA.- $J$. BUON, 11, 2006, No 3, 291-297.

9. Kornovski, Y., G. Gorchev, M. Yakov. Therapeutic approaches to locally advanced cervical cancer.- Akush. ginekol. (Sofia), 2006, No 3, 20-24 (in Bulgarian).

10. Kornovski, Y., G. Gorchev. Histopathological findings in postoperative specimens in cervical cancer patients with stages IB2-IVA after neoadjuvant chemotherapy and preoperative plus postoperative radiotherapy.- J. BUON, 12, 2007, No 1, 57-63.

11. Kornovski, Y., E. Ismail, M. Kaneva. Treatment of cervical cancer in stage IB2.- Akush. ginekol. (Sofia), 2012, No 4, 8-23 (in Bulgarian).

12. Kornovski, Y., E. Ismail, M. Kaneva. Oncological results in the combined treatment of patients with cervical cancer in FIGO stage IIB.- Akush. ginekol. (Sofia), 2012, No 4, 24-29 (in Bulgarian).

13. Monk, B. J., K. S. Tewari. Invasive cervical cancer.- In: Clinical gynecologic oncology. Mosby Elsevier, 2007, 55-124.

14. Quinn, M. A., J. L. Benedet, F. Odicino, P. Maisonneuve, U. Beller, W. T. Creasman, et al. Carcinoma of the cervix uteri. FIGO $26^{\text {th }} \mathrm{An}$ nual Report on the Results of Treatment in Gynecological Cancer.- Int. J. Gynaecol. Obstet., 95, 2006, Suppl. 1, S43-S103. 